\title{
TITLE:
}

\section{On evaluation of certain limits in closed form}

AUTHOR(S):

Kanemitsu, S.

\section{CITATION:}

Kanemitsu, S.. On evaluation of certain limits in closed form. 数理解析 研究所講究録 1985, 572: 8-26

ISSUE DATE:

1985-11

URL:

http://hdl.handle.net/2433/99193

RIGHT: 
On evaluation of certain limits in closed form S. Kanemitsu (金光垪) (Kyushu Univ.)

1. Since Apéry's proof of $\zeta(3) \notin \mathbb{Q}$ or rather van der Poorten's observation that Apéry's series for $\zeta(3)$ has its genesis in the trilogarithm $\mathrm{Li}_{3}(\mathrm{z})$, the theory of irrational numbers as well as the theory of polylogarithms $L i_{\nu}(z)$ are in revival, the latter of whichis also in vogue $(v=2)$ in an algebraic geometrical aspect. I will present here yet another application of polylogarithms, which, setting aside opinions of others, I hope will serve for facilitating the mood of progress in both of these theories. That is, I will apply the polylogarithm of complex exponential argument to evaluate the following three kinds of limits in closed form:

(i) $L^{(k)}(1, x)=(-1)^{k} \sum_{n=1}^{\infty} \frac{x(n) \operatorname{Iog}^{k} n}{n}$, the $k$-th derivative of Dirichlet's L-function $L(s, x)$, evaluated at $s=1$, where $x \neq x_{0}$ is any primitive Dirichlet character $\bmod q, \quad 1<q \in \mathbf{N}, 0 \leq k \in \mathbf{Z}$.

$$
\gamma_{k}(r, q)=\lim _{x \rightarrow \infty}\left(\sum_{n \equiv r(\bmod q)} \frac{\log ^{k} n}{n}-\frac{\log ^{k+1} x}{q(k+1)}\right) \text {, the } k-t h
$$

generalized Euler constant mod $q$ with $q, k$ as above (or rather jokingly, the k-th generelized generalized Euler constant, where the first "generalized" refers to the generalization of the ordinary k-th generalized Euler constant $\gamma_{k}=\gamma_{k}(0,1)$ to the arithmetic progression $r \bmod q)$, where $1<q \in \mathbb{N}$ and we may restict ourselves to $0<r \leq q-1$, since for $r \equiv 0(\bmod q)$, we have 


$$
q \gamma_{k}(0, q)=\gamma_{k}-\log q
$$

and

(iii) $L(k, x)$, the special values of Dirichlet's I-function at positive integral arguments $k$, for non-principal $X$.

Let me first state some historical overview on (i)-(iii). (i), in particular, the evaluation of $L(1, x)$ and $L^{\prime}(1, x)$ in closed form has a long history (of course, (i) and (iii) amounts to the same if $k=1)$. Expressing $L(1, x)$ in terms of a finite sum makes up the second stage of Dirichlet's work on his class number formula, the first stage consisting in relating $L(1, x)$ to the class number of quadratic forms (or equivalently, to that of quadratic fields) of given discriminant, and can be found in many textbooks on number theory (e.g. in Davenport, Multiplicative number theory; Narkiewicz, Elementary and analytic theory of algebraic numbers, or Hasse, Über die Klassenzahl abelscher Zahlkörper). Observe that all existing proofs (as far as I know) depend on Abel's continuity theorem (and a fortiori on the convergence of the power series $\sum_{n=1}^{\infty} \frac{z^{n}}{n}$ on $|z|=1$, $z \neq-1$ ). I will state one more (more or less known) proof of the finite expression of $L(1, x)$, later.

Regarding the finite expression for $L^{\prime}(1, X)$, it was first obtained by Berger in 1883 and independently by Lerch in 1897 for odd characters (see [Lan 1]). Both authors used Kummer's series for $\log \Gamma(x)$, i.e.

(1.1) $\log \Gamma(x)=\frac{1}{\pi} \sum_{n=1}^{\infty} \frac{\sin 2 \pi n x \log n}{n}-\frac{1}{2} \log \frac{\sin \pi x}{\pi}-(\gamma+\log 2 \pi) B_{1}(x)$ valid for $0<x<1$ 
However, for even $x$, the evaluation of $L^{\prime}(1, x)$ led to the evaluation of the Fourier cosine-series

$$
\Phi_{1}(x)=\sum_{n=1}^{\infty} \frac{\log n \cos 2 \pi n x}{n}
$$

which, unlike the sine-series, was not known to be related to any classical function of analysis. After the works of Lerch [Le 1] and Landau [Lan 1], Gut first took up the problem of evaluating $L^{\prime}(1, X)$ for all $X$ by constructing an infinite series (involving squares of logarithms) whose Fourier series coincides with $\Phi_{1}$, to obtain the Kronecker limit formula for the zeta-function of cyclotomic fields. Very recently, Deninger [De], in the spirit of Artin, has developed the theory of R-functions, which provides us with a better understanding of $\Phi_{1}$, where the function $R(x)$ is characterized as in the Bohr-Mollerup theorem (on $\log \Gamma$ ). And it is this standpoint of Deninger (-Artin), combined with Berndt's consideration (cf. [Be]) in our case, that I will adopt in this note.

Finally, regarding (iii), I mention only three references [Ya], [Lew] and [Mi]. Yamamoto seems to be the first to have evaluated $L(k, x)$ in finite form, who, however, did not use the relation (2.17) between $F(s, z)=\sum_{n=1}^{\infty} \frac{e^{2 \pi i n z}}{n^{s}}$ and the Hurwitz zeta-function, but used the finite expression for $F(k, x) \quad(k \in \mathbb{N}, 0<x<1)$ :

$$
F(k, x)=\frac{(2 \pi i)^{k-1}}{k !}\left[A_{k}(x)-\pi i B_{k}(k)\right]
$$

where $A_{k}(x)$ is essentially the repeated integral of the log-sine (or the Clausen) integral 


$$
-\int_{0}^{2 \pi x} \log \left|2 \sin \frac{\theta}{2}\right| d \theta \quad\left(=\mathrm{Cl}_{2}(2 \pi x)\right)
$$

and is the same as the one called the Clausen function in [Lew], and where $B_{k}(x)$ is the $k$-th Bernoulli polynomial defined by

$$
\frac{t e^{x t}}{e^{t}-1}=\sum_{k=0}^{\infty} B_{k}(x) \frac{t^{k}}{k !}
$$

He deduced the Fourier series of $A_{k}(x)$ and $B_{k}(x)$ from the definition of $F(k, x)$. There is, however, a more well-known procedure, which has long been known (see [Lew]), leading to (1.2). It starts with defining the Fourier series themselves as the Clausen functions: $\mathrm{CI}_{2 \mathrm{n}}(\theta)=\sum_{k=1}^{\infty} \frac{\sin \mathrm{k} \theta}{k^{2 n}} ; \mathrm{CI}_{2 \mathrm{n}+1}(\theta)=\sum_{k=1}^{\infty} \frac{\cos k \theta}{k^{2 n+1}}$, and then deducing

$$
\left\{\begin{array}{l}
C I_{2 n+1}(\theta)=L i_{2 n+1}(1)-\int_{0}^{\theta} C I_{2 n}(\theta) d \theta \quad\left(L i_{2 n+1}(1)=\zeta(2 n+1)\right) \\
C I_{2 n}(\theta)=\int_{0}^{\theta} C I_{2 n-1}(\theta) d \theta .
\end{array}\right.
$$

For this and for the associated Clausen functions, see [Lew, p.191几].

2. As we stated in §1, our principal aim is the evaluation of three kinds of limits in finite form. The principle which underlies such an evaluation is fairly simple and depends upon the following classical relation (cf. [Whi-Wa, p.275] and [De,p.176])

$$
\text { (2.1') } F(s, z):=\sum_{n=1}^{\infty} \frac{e^{2 \pi i n z}}{n^{s}}=i \frac{\Gamma(1-s)}{(2 \pi)^{1-s}}\left(e^{-\pi s i / 2} \zeta(1-s, z)-e^{\pi s i / 2} \zeta(1-s, 1-z)\right)
$$

where the first series converges absolutely for $\mathbf{s} \boldsymbol{E} \mathbb{C}, \therefore \operatorname{Im} z>0$ as well as for $\sigma>1, z \in \mathbb{R}$, whereas the right-hand side expression represents an integral function of $s$ whenever $z \notin \mathbb{Z}$. For $z \in \mathbb{Z}$,

$$
-4-
$$


(2.1') should be interpreted as meaning the well-known Riemann's functional equation in its unsymmetric form

(2.2) $\quad F(s, z)=\sum_{n=1}^{\infty} \frac{1}{n^{s}}=\zeta(s)=\frac{2 \Gamma(1-s)}{(2 \pi)^{1-s}} \sin \frac{\pi s}{2} \zeta(1-s)$.

Although (2.11) has long been known, it is nothing but the relation between two independent bases of the vector space $\mathcal{H}_{\mathrm{S}}$ consisting of all continuous maps $f:(0,1) \rightarrow \mathbb{C}$ satisfying the Kubert identity $\left(*_{s}\right)$ (for this and many further interesting results, see [Mil]). Because we shall need the information on $F(s, z)$ only for $0<z<1$, we suppose, in what follows, that $z=x \in(0,1)$.

Now, the fundamental idea (which is, according to [De], due to Meyer) is to use the coefficient of $s^{k}$, or of $s-k$, as the case may be, in the expansion of the right-hand side of (2.1') in order to get an explicit expression of the series

$$
\sum_{n=1}^{\infty} \frac{e^{2 \pi i n j / q} \log ^{k} n}{n}=(-1)^{k} \frac{\partial^{k}}{\partial s^{k}} F\left(1, \frac{j}{q}\right) \quad \text { or } \quad \sum_{n=1}^{\infty} \frac{e^{2 \pi i n x}}{n^{k}}=F(k, x)
$$

in terms of certain definite special functions, which then yields elegant expressions for $L^{(k)}(1, x)$ (and $\gamma_{k}(r, q)$ ) and for $L(k, x)$, $x$ being any Dirichlet chatacter mod $q, q>1$. For notational convenience we shall use (2.1') with $s$ in place of $1-s$ as in [De]:

(2.1) $\quad F(1-s, x)=\frac{\Gamma(s)}{(2 \pi)^{s}}\left(e^{\pi s i / 2} \zeta(s, x)+e^{-\pi s i / 2} \zeta(s, 1-x)\right)$.

The coefficient of $s^{k}\left(=(-1)^{k} \frac{\partial^{k}}{\partial s^{k}} F(1, x)\right)$ is 
(2.3) $\sum_{a+b+c+d=k+1} \frac{(-\log 2 \pi)^{a}}{a !} \frac{\Gamma^{(b)}(1)}{b !} \frac{(\pi i / 2)^{c}}{c ! d !}\left\{\zeta^{(d)}(0, x)+(-1)^{c} \zeta^{(d)}(0,1-x)\right\}$,

where $a, b, c, d$ run through all integers $\geq 0$ whose sum is $k+1$. In actual calculation, one can omit those terms which correspond to $d=0,2 \mid c$, since $\zeta(0, x)+\zeta(0,1-x)=0$.

Thus, for $k=0$, we obtain

$$
F(1, x)=\pi i\left(\frac{1}{2}-x\right)-\log (2 \sin \pi x)
$$

which is formula (2.7.1) of [De] and coincides with the case $k=1$ of Proposition 3.2, (i) of [Ya].

For $k \geq 1$ we already need a new function defined as follows. By [De,p.173] there is a solution $R_{\alpha}(x)$ of the difference equation (whose right-hand side member is a continuous function for $x \geq b>0$ )

$$
f(x+1)-f(x)=\log ^{\alpha} x, \quad 0 \leq \alpha \in Z,
$$

given by (the Gaussian representation)

$$
R_{\alpha}(x)=\lim _{n \rightarrow \infty}\left(\lambda+x \log ^{\alpha} n-\log ^{\alpha} x-\sum_{\nu=1}^{n-1}\left(\log ^{\alpha}(x+v)-\log ^{\alpha} v\right)\right)
$$

such that $R_{\alpha}(x)$ is convex on some interval $(A, \infty), A>0$, and $\mathrm{R}_{\alpha}(1)=\lambda$. Moreover by Theorem $(2.2)$ of $[\mathrm{De}],(-1)^{\alpha+1}\left(\frac{\partial^{\alpha}}{\partial s^{\alpha}} \zeta(0, x)-\zeta^{(\alpha)}(0)\right)$ is the uniquely determined solution of (2.5) having value 0 at $x=1$. (Hence, in particular, for $\alpha=1, \log \Gamma(x)$ is the uniquely determined solution with $\log \Gamma(1)=0$, convex for large argument, since $\partial_{S} \zeta(0, x)$ $=\log \Gamma(x)+\zeta^{\prime}(0)$, and so Theorem (2.2) of [De] is a generalization 
of the well-known Bohr-Mollerup theorem).

Hence

$$
R_{\alpha}(x)=(-1)^{\alpha+1} \frac{\partial^{\alpha}}{\partial s^{\alpha}} \zeta(0, x)
$$

on choosing $\lambda=(-1)^{\alpha+1} \zeta(\alpha)(0)$.

(2.6) can be transformed, by using the definition of $\gamma_{\alpha-1}$,

$$
\gamma_{\alpha-1}=\lim _{n \rightarrow \infty}\left(\sum_{\nu=1}^{n-1} \frac{\log ^{\alpha-1}}{v}-\frac{\log ^{\alpha} n}{\alpha}\right)
$$

(cf. $\$ 1,($ ii)), into

$$
\begin{aligned}
R_{\alpha}(x) & =(-1)^{\alpha+1} \zeta^{(\alpha)}(0)-\gamma_{\alpha-1} x-\log ^{\alpha} x-\sum_{\nu=1}^{\infty}\left(\log ^{\alpha}(x+v)\right. \\
& \left.-\log ^{\alpha} v-\alpha x \frac{\log ^{\alpha-1} v}{v}\right),
\end{aligned}
$$

the Weierstrass representation. The derivatives of $R_{\alpha}$ are then given by

$$
R_{\alpha}^{\prime}(x)=-\gamma_{\alpha-1}-\alpha \frac{\log ^{\alpha-1} x}{x}-\alpha \sum_{\nu=1}^{\infty}\left(\frac{\log ^{\alpha-1}(x+\nu)}{x+\nu}-\frac{\log ^{\alpha-1} \nu}{\nu}\right),
$$

and for $k \geq 2$

$$
R_{\alpha}^{(k)}(x)=\sum_{r=1}^{k} c_{\alpha-r}(k)\left(x^{-k} \log ^{\alpha-r} x-\sum_{\nu=1}^{\infty}(x+\nu)^{-k} \log ^{\alpha-r}(x+\nu)\right)
$$

which are continuous for $x \geq b>0$. Hence, as $x \rightarrow \infty, R_{\alpha}(x) \rightarrow 0$, and this characterizes $R_{\alpha}(x)$ as the Hauptlösung $F(x \mid 1)$ of $(2.5)$ by p.56 of [Nör]. In particular, $R_{2}(x)=R(x)$ (cf.[De]). Observe that although [Be2] gives a Hauptlösung to $\phi(x)-\phi(x-1)=\log ^{2} x$, it is 
not a Hauptlösung of $(2.5)$.

Now we are in a position to evaluate first a few $(-1)^{k} \frac{\partial^{k}}{\partial s^{k}} F(1, x)$. The substitution $\zeta(0, x)=\frac{1}{2}-x, \zeta^{\prime}(0, x)=\log \Gamma(x)-\frac{1}{2} \log 2 \pi, \zeta^{\prime \prime}(0, x)=$ $=-R(x)$ into (2.3) gives

$$
\begin{aligned}
-\frac{\partial}{\partial s} F(1, x) & =-(\gamma+\log 2 \pi)\left(-\log 2 \sin \pi x+\pi i\left(\frac{1}{2}-x\right)\right) \\
& -\frac{1}{2}(R(x)+R(1-x))+\frac{\pi i}{2}(\log \Gamma(x)-\log \Gamma(1-x))
\end{aligned}
$$

or $\quad=-(\gamma+\log 2 \pi) F(1, x)-\frac{1}{2}(R(x)+R(1-x))+\frac{\pi i}{2}(\log \Gamma(x)-\log \Gamma(1-x))$,

which is formula (2.7.2) of [De]. Note that the imaginary parts of both sides give Kummer's series (1.1) for $\log \Gamma(x)$.

Similarly, we obtain

$$
\begin{aligned}
(2.10) & \frac{\partial^{2}}{\partial s^{2}} F(1, x)=\frac{1}{6}\left(R_{3}(x)+R_{3}(1-x)\right)+\frac{1}{2}(\log 2 \pi+\gamma)(R(x)+R(1-x)) \\
& -\frac{\pi i}{4}(R(x)-R(1-x))+\left(\frac{\log ^{2} 2 \pi}{2}+\frac{\zeta(2)+\gamma^{2}}{2}+\gamma \log 2 \pi-\frac{\pi^{2}}{8}\right)(-\log 2 \sin \pi x) \\
& -\frac{\pi i}{2}(\log 2 \pi+\gamma)(\log \Gamma(x)-\log \Gamma(1-x)) \\
& +\pi i\left(\frac{\log ^{2} 2 \pi}{2}+\frac{\zeta(2)+\gamma^{2}}{2}+\gamma \log 2 \pi-\frac{\pi^{2}}{24}\right)\left(\frac{1}{2}-x\right),
\end{aligned}
$$

and so on.

3. We are now in a position to give main formulas in this note. The following relation plays a basic role in the evaluation of $L^{(k)}(1, x)$ as well as of $L(k, X)$ :

$$
L^{(k)}(s, x)=\tau(\bar{x})^{-1} \sum_{j=0}^{q-1} \bar{x}(j) \frac{\partial^{k}}{\partial s^{k}} F\left(s, \frac{j}{q}\right)
$$


valid for $k=0,1,2, \ldots$ and $\sigma>1$, where $\tau(\bar{x})=\sum_{j=0}^{q-1} \bar{x}(j) \varepsilon_{j}(1)$ $\left(\varepsilon_{j}(1)=e^{2 \pi i j / q}\right)$ is the normalized Gauss sum. (3.1) holds for any $s \in \mathbb{C}$ by analytic continuation, and can be proved on the basis of the well-known relation

$$
x(n)=\tau(\bar{x})^{-1} \sum_{j=0}^{q-1} \bar{x}(j) \varepsilon_{j}(n),
$$

where $\varepsilon_{j}(n)=e^{2 \pi i j n / q}$ is an additive character modq. For more details on (3.1), see [Mil] and [Ya].

Although we can transfer the results on $L^{(k)}(1, x)$ onto $\gamma_{k}(r ; q)$ using the relation (cf:[Kno])

$$
L^{(k)}(1, x)=(-1)^{k} \sum_{j=1}^{q} x(j) \gamma_{k}(j, q) \text {, }
$$

we shall adopt the Fourier analysis to $\gamma_{k}(r, q)$ directly as follows. Consider the finite Fourier series (as in [Lel])

$$
\sigma_{k, j}:=\sum_{\lambda=0}^{q-1} \gamma_{k}(\lambda, q) \varepsilon_{\lambda}(1)
$$

for $j \neq 0(\bmod q)$. By the definition of $\gamma_{k}(r, q)$ and the orthogonality relation of $\varepsilon_{\lambda}$, we get

$$
\sigma_{k, j}=(-1)^{k} \frac{\partial^{k}}{\partial s^{k}} F\left(1, \frac{j}{q}\right) .
$$

As noted by [Ya], $\varepsilon_{0}, \varepsilon_{1}, \ldots \varepsilon_{q-1}$ form an orthogonal basis of $\mathrm{C}(\mathrm{q})$, the vector space over $\mathbb{C}$ of all sequences of period $q$, with 
respect to the inner product $\left(c_{1}, c_{2}\right)=\sum_{a \bmod q} c_{1}(a) \bar{c}_{2}(a)$, it follows from (3.2) that

$$
q \gamma_{k}(r, q)=\gamma_{k}+\sum_{j=1}^{q-1}(-1) \frac{\partial^{k}}{\partial s^{k}} F\left(1, \frac{j}{q}\right) \exp (-2 \pi i j r / q)
$$

since $\sigma_{k, 0}=\sum_{j=0}^{q-1} \gamma_{k}(j, q)=\gamma_{k}$.

It remains to substitute $(2.4),(2.8),(2.9),(2.10), \ldots$ into (3.1) and (3.3), respectively.

Regarding $I^{(k)}(1, x)$, the results are:

(3.4) $\quad L(1, x)=\left\{\begin{array}{ll}\pi i \frac{\tau(x)}{q} B_{1}, \bar{x}, & x \text { odd } \\ \frac{\tau(x)}{q} \sum_{j=0}^{q-1} \bar{x}(j) \log \sin \pi \frac{i}{q}, & x \text { even }\end{array}\right.$,

where $B_{1, x}=\sum_{j \bmod q} x(j) \bar{B}_{1}\left(\frac{i}{q}\right)$;

(3.5) $\quad L^{\prime}(1, x)= \begin{cases}\pi i \frac{\tau(x)}{q}\left((\gamma+\log 2 \pi) B_{1}, \bar{x}+\sum_{j=0}^{q-1} \bar{x}(j) \log \Gamma\left(\frac{j}{q}\right)\right), & x \text { odd } \\ -\frac{\tau(x)}{q}\left((\gamma+\log 2 \pi) \sum_{j=0}^{q-1} \bar{x}(j) \log \mid 1-e^{2 \pi i j / q},\right. & x \text { even } \\ -\frac{\tau(x)}{q} \sum_{j=0}^{q-1} \bar{x}(j) R\left(\frac{j}{q}\right), & \end{cases}$

Deninger's formulas (3.4) and (3.6) respectively;

(3.6) $\quad L^{\prime \prime}(1, X)$

$$
\begin{gathered}
=\tau(\bar{\chi})^{-1}\left[-\frac{\pi i}{2} \sum_{j=0}^{q-1} \bar{x}(j) R\left(\frac{j}{q}\right)-\pi i(\log 2 \pi+\gamma) \sum_{j=0}^{q-1} \bar{x}(j) \log \Gamma\left(\frac{j}{q}\right)\right. \\
\left.-\pi i\left(\frac{\log ^{2} 2 \pi}{2}+\frac{\zeta(2) \pm \gamma^{2}}{2}+\gamma \log 2 \pi-\frac{\pi^{2}}{24}\right) B_{1}, \bar{x}\right], \\
-10-
\end{gathered}
$$




$$
\begin{aligned}
& =\tau(\bar{x})^{-1}\left[\frac{1}{3} \sum_{j=0}^{q-1} \bar{x}(j) R_{3}\left(\frac{j}{q}\right)+(\log 2 \pi+\gamma) \sum_{j=0}^{q-1} \bar{x}(j) R\left(\frac{j}{q}\right)\right. \\
& \left.\left.-\left(\frac{\log ^{2} 2 \pi}{2}+\frac{\zeta(2)+\gamma^{2}}{2}+\gamma \log 2 \pi-\frac{\pi^{2}}{8}\right) \sum_{j=0}^{q-1} \bar{x}(j) \log 2 \sin \pi \frac{j}{q}\right)\right], \chi \text { even, }
\end{aligned}
$$

and so on.

\section{Similarly,}

(3.7) $q \gamma_{0}(r, q)=\gamma-\frac{\pi}{2 q} \sum_{j=1}^{q-1}(2 j-q) \sin \frac{2 \pi r j}{q}-\sum_{j=1}^{q-1} \cos \frac{2 \pi r j}{q} \log \sin \frac{\pi j}{q}$

$$
\begin{aligned}
& -(\log 2) \sum_{j=1}^{q-1} \exp (-2 \pi i r j / q) \\
& = \begin{cases}\gamma+\log 2+\frac{\pi}{2} \cot \frac{\pi r}{q}-2 \sum_{1<j<q / 2} \cos \frac{2 \pi r j}{q} \log \sin \frac{\pi j}{q}, & r \equiv 0(q) \\
\gamma-\log q, & r \equiv 0(q),\end{cases}
\end{aligned}
$$

by Lemma A, (c) of [Leh]. (3.7) is the same as formula (11) of [Leh]. Incidentally, as was shown by $[\mathrm{Br} 2]$ and [Leh]

$$
q \gamma_{0}(r, q)=-\psi\left(\frac{r}{q}\right)-\log q
$$

where $\psi=\frac{\Gamma^{\rho}}{\Gamma}$ is Gauss' digamma function. Comparing (3.7) and (3.8) gives Gauss' formula (as in [Leh])

(3.9) $\psi\left(\frac{r}{q}\right)=-r-\log 2 q-\frac{\pi}{2} \cot \frac{\pi r}{q}+2 \sum_{1 \leq j<q / 2} \cos \frac{2 \pi r j}{q} \log \sin \frac{\pi j}{q}$.

$$
\text { For } k=1 \text {, }
$$


(3.10) $q \gamma_{1}(r, q)=\gamma_{1}-(\gamma+\log 2 \pi)\left(q \gamma_{0}(r, q)-\gamma_{0}\right)+\pi \sum_{j=1}^{q-1} \log \Gamma\left(\frac{j}{q}\right) \sin 2 \pi r \frac{j}{q}$

$$
-\sum_{j=1}^{q-1} R\left(\frac{j}{q}\right) \cos 2 \pi r \frac{j}{q} \text {. }
$$

For $\mathrm{k}=2$,

(3.11) $q \gamma_{2}(r, q)=\gamma_{2}-\frac{1}{3} \sum_{j=1}^{q-1} R_{3}\left(\frac{j}{q}\right) \cos 2 \pi j \frac{r}{q}-\frac{\pi}{2} \sum_{j=1}^{q-1} R\left(\frac{j}{q}\right) \sin 2 \pi j \frac{r}{q}$

$$
\begin{aligned}
& -(\log 2 \pi+\gamma)\left(q \gamma_{1}(r, q)-\gamma_{1}\right) \\
& +\left(\frac{3}{2}(\log 2 \pi+\gamma)^{2}+\frac{\zeta(2)}{2}-\frac{\pi^{2}}{8}\right)\left(q \gamma_{0}(r, q)-\gamma\right)+\frac{\pi^{3}}{24} \cot \pi \frac{j}{q},
\end{aligned}
$$

etc.

We conclude this section by considering briefly. $L(k, \chi)$, clinging still to our method. In this case we need to calculate

$$
\zeta^{\prime}(1-k, x)-(-1)^{k} \zeta^{\prime}(1-k, 1-x)
$$

because the coefficient of $s-k$ is

(3.13) $\frac{(2 \pi i)^{k-1}}{k !}\left[k\left(\zeta^{\prime}(1-k, x)-(-1)^{k} \zeta^{\prime}(1-k, 1-x)\right)-\pi i B_{k}(x)\right]$,

which coincides with (2.4) in the case $k=1$, in view of $\zeta^{\prime}(0, x)$ $=\log \Gamma(x)-\frac{1}{2} \log 2 \pi$. By $[B e-J o],(3.12)$ is equal to

$$
\phi_{k-1}(x-1)-\phi_{k-1}(-x), \quad k \text { even }
$$

and to

$$
\phi_{k-1}(x-1)+\phi_{k-1}(-x)+2 \zeta^{\prime}(1-k), \quad k \text { odd }
$$


in their notation. Applying Entry 30, we conclude that

$$
\zeta^{\prime}(1-k, x)-(-1)^{k} \zeta^{\prime}(1-k, 1-x)= \begin{cases}\frac{(k-1) !}{(2 \pi)^{k-1}} \cos \frac{k-1}{2} \pi \sum_{n=1}^{\infty} \frac{\cos 2 \pi n x}{n^{k}}, & k \text { odd } \\ \frac{(k-1) !}{(2 \pi)^{k-1}} \sin \frac{k-1}{2} \pi \sum_{n=1}^{\infty} \frac{\sin 2 \pi n x}{n^{k},} & k \text { even }\end{cases}
$$

Hence substituting this in (3.12), after rewriting the right-hand side members in Lewin's notation introduced at the end of $\$ 1$, we get

$$
F(k, x)=\frac{(2 \pi i)^{k-1}}{k !}\left[(-1)^{(k-1) / 2} \frac{k !}{(2 \pi)^{k-1}} C I_{k}(2 \pi x)-\pi i B_{k}(x)\right]
$$

which is the same as (1.2).

4. Remarks and problems. Defining the function $g(x)$ as in [Br 2] by

$$
\begin{aligned}
& \psi(x)=-\gamma-\frac{1}{x}+g(x) \\
& g(x)=\sum_{n=1}^{\infty} \frac{x}{n(n+x)},
\end{aligned}
$$

we deduce from (3.8)

$$
q \gamma_{0}(r, q)=\gamma+\frac{q}{r}-\log q-g\left(\frac{r}{q}\right), \quad 0<r<q .
$$

Substituting (3.9) in (4.1), we get

(4.4) $g\left(\frac{r}{q}\right)=\frac{q}{r}-\log \frac{q}{2}-\frac{\pi}{2} \cot \frac{\pi r}{q}+2 \sum_{1 \leq j<q / 2} \cos \frac{2 \pi r j}{q} \log \sin \frac{\pi j}{q}$.

To the right-hand side of (4.4) Uchiyama applies Baker's theorem 
that any non-zero linear combination of (finitely many) logarithms of algebraic numbers with algebraic coefficients is transcendental, to conclude that $g(x)$ is transcendental for $0<x<1$, since for such $x, g(x)<1<1 / x$, and a fortiori that

$$
\gamma-q \gamma_{0}(r, q)-\log q
$$

is transcendental. However, the presence of log $q$ in this assertion is a formidable deficiency, and Briggs used the (rather trivial) relation

$$
a q \gamma_{0}(a r, a q)+\log a=q r(r, q)
$$

valid for a $\in \mathbb{N}$, to cancel this $\log q$. (cf. Theorems 2 \& in [Br 2]). It is rash to hazard a conjecture on the transcendency (or even irrationality) of $\gamma_{k}(r, q)$ in the present circumstance that nothing is known even about the irrationality of $\gamma_{k}$, but it does not seem unreasonable to expect the truth of the following:

Problem 1. Is there a non-trivial transcendental (or rational) correlation between $\gamma_{k}(r, q)$ and $\gamma_{k}$ ?

That a generating function is known only for $\gamma_{0}(r, q)$ is not satisfactory at all, and we state

Problem 2. Find a generating function for $\gamma_{k}(r, q)$ and in general transfer Matsuoka's results $\left[\right.$ Ma 1-3] on $\gamma_{k}$ to $\gamma_{k}(r, q)$. As a final problem, we state

Problem 3. Investigate further analogues of the Chowla-Selberg type relation. In other words, examine the arithmetic nature of solutions of the difference equation (2.5).

$$
-14-
$$


A list of papers on the generalized Euler constants

[Be 1] B. C. Berndt, On the Hurwitz zeta-function, Rocky Mountain J. Math. 2(1972), 151-157.

[Be 2] B. C. Berndt, Chapter 8 of Ramanujan's second notebook, J. Reine Angew. Math: 338 (1983), 1-55.

[Be 3] B. C. Berndt, The gamma function and the Hurwitz zeta-function, to appear in Amer. Math. Monthly.

[Br1] W. E. Briggs, Some constants associated with the Riemann zeta-function, Michigan Math.J.3 (1955-56), 117-121.

[Br 2] W. E. Briggs, The irrationality of $\gamma$ or of sets of similar constants, Norske Vid. Selsk. Forh. (Trondheim) 34 (1961), $25-28$.

[ $\mathrm{Br} 3$ ] W.E. Briggs, Some abelian results for Dirichlet series, Mathematika $9(1962), 49-53$.

[Br-Bu] W. E. Briggs and R. G. Buschman, The power series coefficients of functions defined by Dirichlet series, Illinois J.Math. $5(1961), 43-44$.

[Br-Ch] W. E. Briggs and S. Chowla, The power series coefficients of $\zeta(\mathrm{s})$, Amer. Math. Monthly 62 (1955), 323-325.

[De] Chr. Deninger, On the analogue of the formula of Chowla and Selberg for real quadratic fields, J. Reine Angew. Math. $351(1984), 172-1.91$.

[E] Y. Eda, A note on the general divisor problem, Sci. Reports Kanazawa Univ. 3 (1955), 5-9. 
[Fe] R.P.Ferguson, An application of Stieltjes integration to the power series coefficients of the Riemann zeta function, Amer. Math. Monthly 70 (1963), 60-61.

[Gr] J.P.Gram, Note sur le calcul de la fonction $\zeta(\mathrm{s})$ de Riemann, Danske Vid. Selsk. Forh. (Kobenhavn), (1895), 303-308.

[GuI M. Gut, Die Zetafunktion, die Klassenzahl und die Kronecker'sche Grenzformel eines beliebigen Kreiskörpers, Comment. Math. Helv. $1(1930), 160-226$.

[Hu] S.Hurwitz, On a class of functions suggested by the zeta function of Riemann, Ann. of Math. (2) 45 (1944), 340-346.

[I] M. I. Israilov, On the principal term in the divisor problem and the power series of the Riemann zeta-function in a neighborhood of its pole, Trudy Mat. Inst. Steklov 142 (1976), 165-173; English transl. Proc. Steklov Inst. Math. 142 (1979), 175-183.

[Je] J.L.W. V. Jensen, Sur la fonction $\zeta(\mathrm{s})$ de Riemann, C. R. Acad. Sci. Paris 104 (1887), 1156-1159.

[Ka] R. Kaneiwa, on the multiplicative partition function, Tsukuba J. Math. 7 (1983), 355-365.

[Kar] J. Karamata, Evaluation élémentaire des sommes typiques de Riesz de certaines fonction arithmétiques, Acad. Serbe Sci. Publ. Inst. Math. $7(1954), 1-40$.

[Klu] J.C. Kluyver, on certain series of Mr. Hardy, Quart.J. Pure Appl. Math. 50 (1927), 185-1.92. 
[Kno] J.Knopfmacher, Generalized Euler constants, Proc. Edinburgh Math. Soc. $21(1978), 25-32$.

[Lan 1] E. Landau, Ueber die zu einem algebraischen Zahlkörper gehörige Zetafunktion und die Ausdehnung der Tschebyschefschen Primzahltheorie auf das Problem der Vertheilung der Primideale, J. Reine Angew. Math. 125 (1903), 64-188.

[Lan 2] E. Landau, Über eine idealtheoretische Funktion, Trans. Amer. Math. Soc. $13(1912), 1-21$.

[Lav 1] A. F. Lavrik, on the principal term in the divisor problem and the power series of the Riemann zeta-function in a neighborhood of its pole, Trudy Mat. Inst. Steklov 142 (1976), 165-173; English translation, Proc. Steklov Inst. Math. 142 (19.79), $175-183$.

[Lav 2] A. F. Lavrik, Laurent coefficients of the generalized zeta -function. Theory of cubature formulas and numerical mathematics, Pap. Conf. Differential equations and numerical mathematics, Novosibirsk 1978, 160-164 (Russian) (1980).

[Leh] D. H. Lehmer, Euler constants for arithmetical progressions, Acta Arith. 27 (1975), 125-142; Selected papers of D. H. Lehmer, vol. II, 591-608, Charles Babbage Research Center, Manitoba 1981 .

[Ma 1] Y. Matsuoka, A function associated with generalized Euler constants, J.Fac. Educ. Shinshu Univ. 44 (1981), 177-180.

[Ma 2] Y. Matsuoka, Generalized Euler constants associated with the Riemann zeta function, Proc. Conf. Number Theory and Combinatorics, Japan 1984, to appear. 
[Ma 3] Y. Matsuoka, on the power series coefficients of the Riemann zeta function, to appear.

[Mit] D. Mitrovic, The sign of some constants associated with the Riemann zeta-function, Michigan Math.J. 9 (1962), 395-397.

[Mo 1 ] Y. Morita, on the Hurwitz-Lerch L-functions, J.Fac.Sci. Univ. Tokyo Sect. IA $24(1977), 29-43$.

[Mo2] Y. Morita, Examples of p-adic arthmetic functions, Proc. intern. sympos. "Algebraic number theory", Kyoto 1976,143-148, JSPS, Tokyo 1977.

[Na] H. Naito, The p-adic Hurwitz L-functions, Tôhoku Math. J. 34 $(1982), 553-558$.

[Scze]. R. Sczech, Zur Summation von L-Reihen, Bonner Math. Schr. $141(1982)$.

[Sta] E. Stanks, on the coefficients of the Laurent expansion of the Riemann zeta-function, Litovsk. Mat. Sb. 23 (1983), 87-88.

[U] S. Uchiyama, An application of a theorem of A. Baker, Proc. sympos. transcendence theory 1983, 91-94, Gakushuin Univ.

[Ve] D. P. Verma, Laurent's expansion of Riemann's zeta function, Indian J. Math. 5 (1963), 13-16.

[Whi-Wa] E.T. Whittaker and G.N. Watson, A course of modern analysis, Cambridge Univ. Press, London 1902.

[Ya]. Y. Yamamoto, Dirichlet series with periodic coefficients, Proc. intern. sympos. "Algebraic number theory", Kyoto 1976, 275-289, JSPS, Tokyo 1977. 


\section{Additional references}

[Be-E] B. C. Berndt and R.J.Evans, Chapter 7 of Ramanujan's second notebook, Proc. Indian Acad.Sci. (Math.Sci.) 92 (1983), 67-96.

[Be-J] B. C. Berndt and P. T. Joshi, Chapter 9 of Ramanujan's second notebook, Contemporary Math. Vol.23, AMS, Providence R.I., 1983.

[Dem] J.-P. Demailly, Sur le calcul numerique de la constante d'Euler.

[Lam] E. Lammel, Eine Beweis, dass die Riemannsche Zetafunktion $\zeta(\mathrm{s})$ in $|\mathrm{s}-1| \leq 1$ keine Nullstelle besitzt, Univ. Nac. Tucumán Rev. Ser. A 16 (1966), 209-217.

[Lew]. Lo Lewin, Polylogarithms and associated functions, North-Holland, New York 1981.

[Ii-T] J.J.Y. Liang and J.Tod, The Stieltjes constants, J.Res. Nat. Bur. Standards Sect. B 76 (1972), 161-178.

[Ma 4] Y. Matsuoka, A note on the relation between generalized Euler constants and the zeros of the Riemann zeta function, J.Fac. Educ. Shinshu Univ. 53 (1985), 81-82.

[Ma 5] Y. Matsuoka, A sequence associated with the zeros of the Riemann zeta function, to appear.

[Mil].. J. Milnor, On polylogarithms, Hurwitz zeta functions, and the Kubert identities, Enseign. Math.(2) 29 (1983), 281-322. 